\title{
Evaluation of skin irritation and skin sensitization potential of Venusia max lotion (paraben-free, alcohol-free, mineral oil-free, animal origin free) using human repeat insult patch test
}

\author{
Monil Y. N. Gala ${ }^{1 *}$, Snehal S. Muchhala ${ }^{1}$, Sujeet N. Charugulla1, Rahul Rathod ${ }^{1}$, Amey Mane ${ }^{1}$, \\ Sucheta Pandit ${ }^{1}$, Alok R. Samal ${ }^{2}$, Anup A. Choudhury ${ }^{2}$
}

\begin{abstract}
${ }^{1}$ Department of Medical Affairs, ${ }^{2}$ Department of Formulation Research and Development, Dr. Reddy's Laboratories Ltd., Hyderabad, India
\end{abstract}

Received: 31 July 2021

Revised: 27 October 2021

Accepted: 28 October 2021

\author{
*Correspondence: \\ Dr. Monil Y. N. Gala, \\ E-mail: Monil.yogesh@drreddys.com
}

Copyright: () the author(s), publisher and licensee Medip Academy. This is an open-access article distributed under the terms of the Creative Commons Attribution Non-Commercial License, which permits unrestricted non-commercial use, distribution, and reproduction in any medium, provided the original work is properly cited.

\begin{abstract}
Background: Topical exposure to chemicals from cosmetics can lead to adverse skin effects or skin irritation. This study aimed to investigate the skin irritation and sensitizing potential of a moisturizer Venusia max lotion (parabenfree, alcohol-free, mineral oil-free, animal origin free (PAMA) free).

Methods: In this single-center, non-randomized, observational study, skin irritation, and skin sensitization potential of a test product was assessed using the human repeat insult patch test (HRIPT) technique. Approximately $0.04 \mathrm{~g}$ of the test product and filter papers dipped in $0.9 \%$ isotonic saline solution $(\sim 0.04 \mathrm{ml}$ of solution) were filled in different wells of patch chambers and applied occlusively, on the back of each participant. Scoring of the skin reactions in the induction phase and challenge phase was done using Draize and international contact dermatitis research group (ICDRG) scales respectively. Scores were compared to the baseline and the negative control (isotonic saline).

Results: In total 234 participants (50 with sensitive skin), 224 and 221 participants completed the induction phase and challenge phase respectively. Scores for the induction phase for Venusia max lotion (PAMA free) and isotonic saline were 0.46 and 0.06 respectively. The mean cumulative score of erythema and oedema for Venusia max lotion (PAMA free) was below 2 . For the challenge phase, none of the participants showed any positive reactions at any time point for test product and isotonic saline.

Conclusions: Test product Venusia max lotion (PAMA free) found to be non-irritant and hypoallergenic. Thus, it can be used without fear of skin irritation or sensitization.
\end{abstract}

Keywords: Moisturizer, Allergic contact dermatitis, Human repeat insult patch test, Irritants, Skin sensitization

\section{INTRODUCTION}

Moisturizers are one the commonly used cosmetic products that are used to maintain good skin health. They are integral in the management of various skin diseases associated with or without skin dryness and those linked to impaired skin barrier function. ${ }^{1,2}$ Even in the management of acne, adjuvant treatments such as skin moisturizers are recommended. ${ }^{3,4}$ The use of moisturizers provides additional anti-inflammatory and skin-soothing effects that may contribute further to overall patient satisfaction. ${ }^{5}$ However, being a cosmetic adjuvant, all moisturizers should be free from allergenicity. Many ingredients present in various moisturizers are identified to be irritants and can cause allergic contact dermatitis (ACD). ${ }^{6}$ In fact, cosmetic and skincare products are said to be the one of important causes of both irritant and allergic reactions. ${ }^{7,8}$ 
Thus, it is important to establish the safety of moisturizers with regard to their irritant and allergic potential. Human testing for the evaluation of the allergenic potential of various compounds is an established standard procedure. European society of contact dermatitis guidelines advocates patch testing to diagnose contact allergy resulting from type IV hypersensitivity. ${ }^{9}$ Thus, the objective of our study was to assess the allergenicity of a moisturizer Venusia max lotion (paraben-free, alcoholfree, mineral oil-free, animal origin free (PAMA) free) using the human repeat insult patch test (HRIPT).

\section{METHODS}

\section{Setting and design}

This was a single-center, non-randomized, observational study to assess the skin irritation and skin sensitization potential of Venusia max lotion (PAMA free). The study was carried out at C.L.A.I.M.S. Pvt. Ltd., Andheri, Mumbai, under the supervision of the principal investigator/dermatologist. Study was conducted from 10 August 2020 to 04 December 2020. The study protocol was approved by institutional ethics committee at C.L.A.I.M.S. Pvt. Ltd., Andheri, Mumbai. The study was conducted respecting the principle of the Declaration of Helsinki and its amendments in conformity with the good clinical practices principles and schedule Y. Potential risks and benefits were explained to the participants and informed consent was obtained from all participants before entry into the study.

\section{Population}

Adult men and women of age 18 to 55 years having apparently healthy skin on the test area, with skin type III to $\mathrm{V}$, were eligible for inclusion in the study. At least 50 participants with sensitive skin as tested by lactic acid sting test were included. Participants who were included in the study agreed to avoid water contact (such as swimming, excessive sweating activity like exercise, and sauna) and avoid intense ultraviolet exposure on the test site during the course of the study. We excluded females who were pregnant or lactating, participants with scars, tattoos, excessive terminal hair on the test area, and those who had previous hypersensitivity (allergy antecedent) to any cosmetic product or hair dyes. Also, participants having a chronic illness that may influence the outcome of the study, participants on any medical treatment either systemic or topical which may interfere with the performance of the study (presently or in the past 1 month), and participants in an exclusion period or participating in another food, cosmetic or therapeutic trial were excluded.

\section{Treatments and follow-ups}

There were two products involved in the study. Test product was Venusia max lotion (PAMA free) (contains shea butter, mango butter, cocoa butter, aloe butter, cetyl alcohol, stearic acid, emulsifying waxes, cyclomethicone, dimethicone, phenoxyethanol, propylene glycol, glycerin, disodium ethylenediaminetetraacetic acid (EDTA), zinc oxide, and purified water). Negative control was $0.9 \%$ isotonic solution of saline.

Patch application was done on the back of participants. Approximately $0.04 \mathrm{~g}$ of the test product and filter papers dipped in $0.9 \%$ isotonic saline solution (to contain approximately $0.04 \mathrm{ml}$ of solution) were filled in different wells of patch chambers and applied occlusively, on the back of each participant by single, trained study personnel. The duration of occlusion was 24 hours. There were two phases of patch application. In phase I (induction phase), patches were applied 9 consecutive times for each subject. On alternate days, the patch area was graded and scored. In phase II (challenge phase), a single patch application was done for 24 hours. Two batches of participants were made randomly. In batch 1 , the challenge was done after a rest of two weeks whereas in batch 2 , the challenge was done after three weeks rest period from the induction phase. In the challenge phase, patch application was done on naïve sites adjacent to the original induction patch sites. The patch was removed after 24 hours, and scoring was done at 48 hours, 72 hours and 96 hours from patch removal.

\section{Scoring}

Scoring of the test area was done with the Draize scale as shown in Table $1 .^{10}$ In the challenge phase, reactions were scored using the international contact dermatitis research group (ICDRG) scale as shown in Table 2. ${ }^{9}$

Table 1: Draize scale scoring system.

\begin{tabular}{|lllll|}
\hline Erythema and eschar formation & & Oedema formation & \\
\hline No reaction & Score 0 & No oedema & Score 0 \\
\hline Very slight erythema & Score 1 & Very slight oedema (barely perceptible) & Score 1 \\
\hline Well-defined erythema & Score 2 & $\begin{array}{l}\text { Slight oedema (edges of area well defined by } \\
\text { definite raising) }\end{array}$ & Score 2 \\
\hline Moderate to severe erythema & Score 3 & Moderate oedema (area raised 1 mm & Score 3 \\
\hline $\begin{array}{l}\text { Severe erythema (beet redness) to slight } \\
\text { eschar formation (injuries in depth) }\end{array}$ & Score 4 & $\begin{array}{l}\text { Severe oedema (raised >1 mm and extending } \\
\text { beyond area of exposure) }\end{array}$ & Score 4 \\
\hline
\end{tabular}


Table 2: International contact dermatitis research group (ICDRG) scale.

\begin{tabular}{|lll|}
\hline Symbol & Morphology & Interpretation \\
\hline- & No reaction & Negative reaction \\
\hline $\mathbf{+ +}$ & Faint erythema only & Doubtful reaction \\
\hline+ & Erythema, infiltration, possibly papules & Weak positive \\
\hline++ & Erythema, infiltration, papules, vesicles & Strong positive \\
\hline $\mathbf{+ + +}$ & Intense erythema, infiltration, coalescing vesicles & Extreme positive \\
\hline $\mathbf{I R}$ & Different types of reactions (soap effect, vesicles, blister, necrosis) & Irritant \\
\hline
\end{tabular}

\section{Adverse effects}

Systemic and local adverse effects (AEs) were monitored in all participants. Any treatment emergent AEs were identified and treated accordingly.

\section{Statistical analysis}

The data from participants was entered in the Microsoft excel spreadsheet 2016 and was analyzed with the same. Descriptive statistics were used to analyze the data. Frequency and percentage was used to present categorical data whereas mean and standard deviation was used to present conmtinuous variables.

\section{RESULTS}

Overall, 234 adults aged 18 to 55 years were enrolled in this study. From them, 224 and 221 participants completed induction and challenge phases respectively. In total, 13 participants dropped out for various reasons. Six and five participants from batch 1 and batch 2 respectively were lost to follow-up. Two participants developed adverse events and thus dropped out of the study.

\section{Demographic characteristics}

Study was conducted between August 2020 and December 2020. In 221 participants enrolled in the study, the mean age was $32.55 \pm 10.53$ years, with a minimum age being 18 years and maximum age being 54 years. The proportion of women and men was $170(77 \%)$ and 51 (23\%). Among participants included, 50 had sensitive skin on the nasolabial area as determined by the lactic acid sting test.

\section{Product assessment score}

Table 3 provides average Draize scale scores for the induction phase after each patch application for Venusia max lotion (PAMA free) and isotonic saline. The scores have been summated for erythema and oedema formation. The average (erythema and oedema) cumulative score for Venusia max lotion (PAMA free) was below 2.

Table 4 shows the ICDRG scale score in the challenge phase. There were no reactions in any of the study participants at 48 hours, 72 hours, and 96 hours after patch removal.
Table 3: Average Draize scale scores for induction phase for two products.

\begin{tabular}{|lll|}
\hline $\begin{array}{l}\text { Patch application } \\
\text { number }\end{array}$ & $\begin{array}{l}\text { Venusia max } \\
\text { lotion (PAMA } \\
\text { free) }\end{array}$ & $\begin{array}{l}\text { Isotonic } \\
\text { saline }\end{array}$ \\
\hline $\mathbf{1}$ & 0.09821 & 0.01339 \\
\hline $\mathbf{2}$ & 0.16518 & 0.03571 \\
\hline $\mathbf{3}$ & 0.20089 & 0.04018 \\
\hline $\mathbf{4}$ & 0.28571 & 0.06250 \\
\hline $\mathbf{5}$ & 0.45089 & 0.04464 \\
\hline $\mathbf{6}$ & 0.53571 & 0.04911 \\
\hline $\mathbf{7}$ & 0.71429 & 0.09821 \\
\hline $\mathbf{8}$ & 0.84375 & 0.09375 \\
\hline $\mathbf{9}$ & 0.85714 & 0.06696 \\
\hline $\begin{array}{l}\text { Average } \\
\text { cumulative score }\end{array}$ & 0.4613 (i.e. 0.46$)$ & $\begin{array}{l}0.05605 \\
\text { (i.e. } 0.06)\end{array}$ \\
\hline
\end{tabular}

Table 4: ICDRG scale score in the challenge phase of two groups.

\begin{tabular}{|l|l|l|}
\hline $\begin{array}{l}\text { Time of } \\
\text { examination after } \\
\text { patch removal }\end{array}$ & $\begin{array}{l}\text { Venusia max } \\
\text { lotion (PAMA } \\
\text { free) }\end{array}$ & $\begin{array}{l}\text { Isotonic } \\
\text { saline }\end{array}$ \\
\hline $\mathbf{4 8}$ hours & - & - \\
\hline $\mathbf{7 2}$ hours & - & - \\
\hline $\mathbf{9 6}$ hours & - & - \\
\hline
\end{tabular}

Symbol (-) indicates no reaction

\section{Adverse events}

Two participants developed AE. Within a half-hour of patch application, headache developed, followed by vomiting and palpitation. AE resolved with symptomatic treatment. Another participant had developed prickly heat on his back because of which patch application was not possible. The erythema from prickly heat subsided after two days without any treatment. Both the participants were discontinued from the study.

\section{DISCUSSION}

Globally, over $75 \%$ of the young population uses moisturizers regularly. It enhances and preserves the smoothness of the skin by improving the skin's hydration and influencing the elasticity of the stratum corneum. ${ }^{12}$ Even in dermatological disorders such as acne, the use of moisturizers is strongly advised along with other topical 
and systemic options. ${ }^{11}$ Though generally considered safe, skin reactions may occur with the use of moisturizers. In evaluating the suspected allergic contact dermatitis due to cosmetics using patch testing, Kumar and Paulose observed a $3.75 \%$ rate of a positive reaction in a total of 2531 patches. Gallate mix $(40 \%)$, cetrimide $(28 \%)$, and thiomersal $(20 \%)$ were the most common allergens identified. Among the products, face creams (20\%), hair dyes (14\%), soaps (12\%), shaving creams (10\%), perfumes $(8 \%)$, and lipsticks $(4 \%)$ were identified as causes for ACD. ${ }^{13}$ Other studies identified fragrance mix as common content of moisturizers that may lead to ACD ${ }^{6,8}$ Cohen et al observed that products such as aveeno, cetaphil, and cerave had a total of 12,14 , and 9 potential allergens respectively which were infrequent and not included in standard patch testing series. ${ }^{14}$ Thus, testing of moisturizers for allergenic potential is advised. Being free from parabens, this lotion has lower risk of irritancy and free from any carcinogenic risks that have been reported with parabens. Also, being free of mineral oil, there is no risk to trapping other pore-clogging ingredients in the skin pores.

Patch testing is an established procedure for assessing the irritant and allergic potential of any product. Patch testing can be performed by various ways such as open patch test, occluded patch test, repeated open application test, semiopen test, and photo patch test. ${ }^{9}$ In this study, we employed HRIPT. It is the most reliable test method by which confirmatory human data can be made available. ${ }^{15} \mathrm{We}$ observed that our moisturizer product Venusia max lotion (PAMA free) did not illicit any irritant or allergenic reaction when studied with HRIPT. In a similar study, Nisbet assessed lamellar moisturizer (containing aqua, butyrospermum parkii butter, caprylic/capric triglyceride, carbomer, ceramide NP, cocos nucifera oil, glycerin, hydrogenated lecithin, hydroxyethylcellulose, pentylene glycol, sodium carbomer, squalane, and xanthan gum) in comparison to negative control of saline. Of 233 participants, 214 completing the study underwent dermatological assessment. They reported negative patch test results in $99.6 \%$ of the participants indicating low allergenic potential of the moisturizer. ${ }^{16}$

As we involved both genders, along with some participants having the sensitive skin, and skin type III to V, results can be applied to the wider population given the low allergenic potential of test product - Venusia max lotion (PAMA free). In addition, being free from parabens and alcohol, this lotion has lower risk of irritancy and skin sensitivity. Also, being free of mineral oil, the risk of trapping other pore-clogging ingredients in the skin pores is non-existent.

\section{Limitations}

The limitations of our study include that we did not assess the allergenicity effect according skin type. Also, we did not compare the results among those with sensitive skin to patients with non-sensitive skin.

\section{CONCLUSION}

In conclusion, test product Venusia max lotion (PAMA free) showed no allergenic potential and can be used without any hesitancy. As none of the participant demonstrated positive results in our human repeat insult patch test, it can be considered for healthy skin as well as in patients with various dermatoses including those with sensitive skin for skin moisturization.

\section{ACKNOWLEDGEMENTS}

Authors would like to thank Dr. Vijay M. Katekhaye (quest MedPharma consultants, Nagpur, India) for his assistance in drafting, editing and reviewing the manuscript.

Funding: The study was funded by Dr. Reddy's Laboratory Ltd., Hyderabad, India

Conflict of interest: All the authors are employees of Dr Reddys Laboratories

Ethical approval: The study was approved by the institutional ethics committee

\section{REFERENCES}

1. Xu S, Kwa M, Lohman ME, Evers-Meltzer R, Silverberg JI. Consumer Preferences, Product Characteristics, and Potentially Allergenic Ingredients in Best-selling Moisturizers. JAMA Dermatol. 2017;153:1099-105.

2. Purnamawati S, Indrastuti N, Danarti R, Saefudin T. The Role of Moisturizers in Addressing Various Kinds of Dermatitis: A Review. Clin Med Res. 2017;15:75-87.

3. Goh CL, Abad-Casintahan F, Aw DC, Baba R, Chan LC, Hung NT, et al. South-East Asia study alliance guidelines on the management of acne vulgaris in South-East Asian patients. J Dermatol. 2015;42:94553.

4. Kubba R, Bajaj AK, Thappa DM, Sharma R, Vedamurthy M, Dhar S, et al. Indian Acne Alliance (IAA). Acne in India: guidelines for management IAA consensus document. Indian $\mathrm{J}$ Dermatol Venereol Leprol. 2009;75:1-62.

5. Chularojanamontri L, Tuchinda P, Kulthanan K, Pongparit K. Moisturizers for Acne: What are their Constituents? J Clin Aesthet Dermatol. 2014;7:3644.

6. Zirwas MJ, Stechschulte SA. Moisturizer allergy: diagnosis and management. J Clin Aesthet Dermatol. 2008; 1:38-44.

7. Held E. So moisturizers may cause trouble! Int J Dermatol. 2001;40:12-3.

8. Goel S, Shetty VH, Eram H, Babu AM. Study of the clinical pattern of contact dermatitis over the face and its correlation with patch testing. Int J Res Dermatol. 2019;5:350-6.

9. Johansen JD, Aalto-Korte K, Agner T, Andersen KE, Bircher A, Bruze M, et al. European Society of 
Contact Dermatitis guideline for diagnostic patch testing-recommendations on best practice. Contact Dermatitis. 2015;73:195-221.

10. Farage MA, Maibach HI, Andersen KE, Lachapelle JM, Kern P, Ryan C, et al. Historical perspective on the use of visual grading scales in evaluating skin irritation and sensitization. Contact Dermatitis. 2011;65:65-75.

11. Dall'oglio F, Tedeschi A, Fabbrocini G, Veraldi S, Picardo M, Micali G. Cosmetics for acne: indications and recommendations for an evidence-based approach. G Ital Dermatol Venereol. 2015;150:1-11.

12. Loden M. Do moisturizers work? J Cosmet Dermatol. 2003;2:141-9.

13. Kumar P, Paulose R. Patch testing in suspected allergic contact dermatitis to cosmetics. Dermatol Res Pract. 2014;695387.

14. Cohen SR, Cárdenas-de la Garza JA, Dekker P, Haidari W, Chisolm SS, Taylor SL, et al. Allergic
Contact Dermatitis Secondary to Moisturizers. J Cutan Med Surg. 2020;24:350-9.

15. McNamee PM, Api AM, Basketter DA, Frank Gerberick G, Gilpin DA, Hall BM, et al. A review of critical factors in the conduct and interpretation of the human repeat insult patch test. Regul Toxicol Pharmacol. 2008;52:24-34.

16. Nisbet SJ. Absence of human skin irritation and allergenic potential after repeated patch applications of a lamellar moisturizer. J Cosmet Dermatol. 2019;18:377-82.

Cite this article as: Gala MYN, Muchhala SS, Charugulla SN, Rathod R, Mane A, Pandit S, et al. Evaluation of skin irritation and skin sensitization potential of Venusia max lotion (paraben-free, alcohol-free, mineral oil-free, animal origin free) using human repeat insult patch test. Int J Res Dermatol 2022;8:26-30. 